\title{
GENERAL FEATURES OF CALCIUM PYROPHOSPHATE DEPOSITION DISEASE
}

\author{
Paulina Vele ${ }^{1}$, Laura Damian², Siao-Pin Simon ${ }^{1,2}$, Ioana Felea ${ }^{1,2}$, Laura Muntean ${ }^{1,2}$, \\ Maria Tamas ${ }^{1,2}$, Simona Rednic ${ }^{1,2}$ \\ ${ }^{1}$ Iuliu Hatieganu University of Medicine and Pharmacy, Cluj-Napoca \\ ${ }^{2}$ Rheumatology, Emergency County Teaching Hospital, Cluj-Napoca
}

\begin{abstract}
Background. Calcium pyrophosphate deposition (CPPD) disease is due to deposition of calcium pyrophosphate to hyaline cartilage, fibrocartilage and soft tissues. Clinical presentation may be as acute arthritis, chronic arthritis and associated with osteoarthritis. CPPD can be idiopathic or secondary, due to different metabolic diseases. Objectives. The aim of this study was to evaluate the clinical characteristics, secondary causes and risk factors of symptomatic CPPD.

Material and methods. Patients diagnosed with CPPD upon presentation for joint pain and/or limitation of motion in Cluj-Napoca Rheumatology Department, were prospectively included in the study. Demographic data, clinical features, laboratory parameters for secondary causes of CPPD and risk factors were recorded.

Results. The study included 40 patients with CPPD. A percentage of $70 \%$ patients presented with acute arthritis. The knee was the most affected joint. During the assessment for secondary CPPD, three patients with hypomagnesaemia and one patient with hypophosphatasia were found. Screening for alkaptonuria was negative in all patients tested (30 patients). Regarding risk factors, $65 \%$ of patients were over 60 years old, osteoarthritis was associated in $80 \%$ of cases, joint injury in $2 \%$, meniscectomy in $6 \%$, diuretics use in $18 \%$, family history of CPPD $2 \%$. Conclusions. Acute arthritis of the knee is the most frequent form of CPPD presentation. Ageing and osteoarthritis are common risk factors for CPPD. Although secondary causes of CPPD are rare, screening is very important.

Keywords: CPPD, clinical features, secondary causes, risk factors
\end{abstract}

\section{INTRODUCTION}

Calcium pyrophosphate deposition (CPPD) disease is a form of inflammatory arthritis due to deposition of calcium pyrophosphate into hyaline cartilage, fibrocartilage and soft tissues. CPPD manifests clinically with a wide range of syndromes. According to the McCarthy classification, the clinical forms are pseudogout, pseudo-rheumatoid arthritis, pseudo-osteoarthritis with and without acute attacks, asymptomatic and pseudoneuropathic (1). The European League Against Rheumatism (EULAR) has recently established the terminology and a new classification. Clinical presentations are acute and chronic CPP crystal arthritis, asymptomatic CPPD and osteoarthritis with CPPD (2).

CPPD is classified as primary, when no causal association is identified, and secondary. The main secondary causes of CPPD are primary hyperparathyroidism, hypomagnesaemia, haemochromatosis, hypophosphatasia and alkaptonuria. There are some established and possible risk factors for CPPD: ageing, joint injury, meniscectomy, osteoarthritis, medication which promote hypomagnesaemia (diuretics, cyclosporine and proton pump inhibitors), chronic kidney disease (2-8).

The standard tool for diagnosis is synovial fluid analysis with identification of CPP crystal by compensated polarized light (9). Radiographic and ultrasonographic evidence of CPPD are used for diagnosis too. The radiographic evidence of CPPD is considered a thin, parallel line with the articular surface (hyaline cartilage calcification) and punctate fibrocartilage calcification in the knees, symphysis pubis, wrists, hips, elbows (10-12). Ultrasonographic diagnosis of CPPD is made by the Frediani's ultrasonographic patterns. There are described three ultrasonographic patterns. The first pattern is described as a thin hyperechoic bands, parallel to the surface of the hyaline cartilage, pattern II - hyperechoic spots and pattern III - hyperechoic nodular or oval deposits in recesses and bursae $(13,14)$. 
CPPD treatment is generally based on clinical experience; there is no treatment to influence the pathogenesis of the disease. Nonsteroidal anti-inflammatory drugs, colchicine and long-acting glucocorticoid injection are the mainstays of the treatment. The aim of the treatment is the resolution of acute arthritis and management of the associated diseases $(15,16)$.

\section{MATERIAL AND METHODS}

The aim of this study was to evaluate the clinical characteristics, secondary causes and risk factors of CPPD in symptomatic patients.

We performed a prospective observational study on consecutive patients with a form of inflammatory arthritis and evidence of CPPD attending the Rheumatology Department, Cluj-Napoca, Romania between January 2015 and July 2016 X-ray Radiography, ultrasonography and, whenever possible, joint aspiration and synovial fluid crystallographic analysis of the symptomatic joints were performed. The radiographic evidence of CPPD is was defined as hyaline cartilage calcification and punctate fibrocartilage calcification in at least one of the affected joints, while Frediani's ultrasound patterns were considered for ultrasonographic evidences of CPPD. Patients with other causes of arthritis were excluded from the study.

Age (years), sex, weight $(\mathrm{kg})$, height $\left(\mathrm{m}^{2}\right)$, body mass index (BMI) $\left(\mathrm{kg} / \mathrm{m}^{2}\right)$, type of joint involvement and pattern of arthritis, as well as disease duration were recorded. Also information about joint trauma, meniscectomy, familial history of CPPD, chronic renal insufficiency, diuretics use, alcohol consumption and comorbidities were registered as well. Patients were tested for erythrocyte sedimentation rate, Creactive protein, blood urea nitrogen, creatinine, total calcium, magnesium, iron, alkaline phosphatase. Also, Furthermore, 30 patients were screened for alkaptonuria using a urine alkalinization test with sodium hydroxide $(\mathrm{NaOH})$. Tests were considered positive if was a change in color of urine with the apparition of a brown ring due to oxidation.

All patients enrolled in the study consented for participation and the approval of the University Ethics Committee was in place for this research.

\section{RESULTS}

The study included 40 patients with CPPD, $80 \%$ females, mean age $63.8 \pm 11.74$ years and a medium BMI of $28.42 \pm 4.37$.

\section{CLINICAL CHARACTERISTICS}

28 patients $(70 \%)$ presented with acute arthritis and 12 patients $(20 \%)$ with chronic arthritis. The majority of patients had oligoarticular (15 patients; $37.5 \%$ ) followed by polyarticular disease (13 patients; 32.5\%) and monoarticular disease (12 patients; $30 \%$ ). The average time since the beginning of symptoms until the presentation was 1.7 weeks. The characteristics of the study population are summarized in Table 1.

TABLE 1. General characteristics of study participants

\begin{tabular}{|l|c|}
\hline Variable & Patients $(\mathrm{n}=40)$ \\
\hline Age (years) & $63.8 \pm 11.74$ \\
\hline Height $(\mathrm{m})$ & $1.65 \pm 0.08$ \\
\hline Weight $(\mathrm{kg})$ & $161 \pm 27.18$ \\
\hline BMI $\left(\mathrm{kg} / \mathrm{m}^{2}\right)$ & $28.42 \pm 4.37$ \\
\hline Sex ratio of male:female & $8: 32$ \\
\hline Tender joint count & $4.125 \pm 3.24$ \\
\hline Swollen joint count & $1.7 \pm 1.17$ \\
\hline Disease duration (weeks) & $1.7 \pm 1.13$ \\
\hline
\end{tabular}

Clinically, the most affected joint clinically was the knee (33 patients), followed by hand (12 patients), shoulder ( 9 patients), ankle (7 patients), hip (3 patients) and elbow (1 patient). In patients without knee involvement, the most affected joint was the hand (4 patients), followed by hip (3 patients) and shoulder (2 patients). Notably, no patient with CPPD - related hip disease had knee involvement.

Synovial fluid samples were obtained in 25 patients; CPP crystals were identified in 23 patients.

Radiographic and ultrasonographic evidence of CPPD was found in all patients, in at least one joint. The knee was the most frequently affected joint, $70 \%$ of patients (30 cases) presenting with both $\mathrm{X}$ ray and ultrasound typical modifications. Distribution of joint involvement by clinical, radiographic and ultrasonographic examination is summarized in Fig. 1. The second Frediani's ultrasonographic pattern was found in 20 patients $(50 \%)$.

\section{Secondary causes}

Patients were screened for secondary causes of CPPD (Table 2). None of our patients had hypercalcemia and haemochromatosis, one patient had hypophosphatasia, and three patients had hypomagnesaemia (two of them under diuretics treatment). Screening for alkaptonuria was negative in all tested patients. 


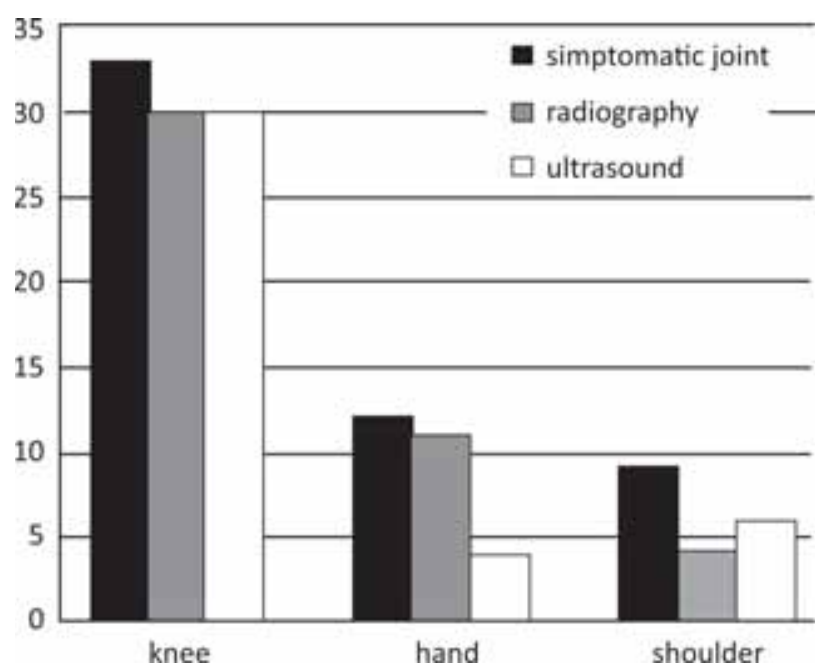

FIGURE 1. Distribution of joint involvment by clinical, radiographic and ultrasonographic examination

TABLE 2. The distribution of patients by CPPD secondary causes

\begin{tabular}{|l|c|}
\hline Secondary causes & Number of patients \\
\hline hyperparathyroidism & 0 \\
\hline haemochromatosis & 0 \\
\hline hypophosphatasia & 1 \\
\hline hypomagnesaemia & 3 \\
\hline alkaptonuria & 0 \\
\hline
\end{tabular}

\section{Risk factors}

Several risk factors for CCPD were demonstrated in our study as follows: joint injury (1 patient, $2 \%$ ), meniscectomy ( 3 patients, $6 \%$ ), diuretics ( 9 patients, $18 \%$ ), family history of CPPD (1 patient, 2\%). Amongst patients on diuretic treatment, seven were under thiazide and two of under loop diuretics. The distribution of patients by risk factors is summarized in Figure 2. From the other possible risk factors, alcohol consumption over 3 units a week was not found in any patient; none had renal insufficiency.
Radiographic evidence of osteoarthritis was observed in 32 patients (80\%), 22.5\% of patients had more than two affected joints. The most affected joint was the knee (27 patients, $67,5 \%$ ) followed by hand ( 8 patients, $20 \%)$, shoulder ( 6 patients, $15 \%)$, hip (3 patients, 7,5\%) and ankle (2 patients, 5\%).

Mean age of patients included in the study is $63.8 \pm 11.74$. The majority of patients were in seven and eight decade. Distribution of patients on age intervals is summarized in Table 3 .

TABLE 3. The distribution of patients by age in number of cases and percentages

\begin{tabular}{|l|c|c|}
\hline Age group & Number of patients & Percentage \\
\hline$<40$ years & 1 & $2.5 \%$ \\
\hline $41-50$ years & 6 & $15 \%$ \\
\hline $51-60$ years & 7 & $17.5 \%$ \\
\hline $61-70$ years & 13 & $32.5 \%$ \\
\hline$>70$ years & 13 & $32.5 \%$ \\
\hline Total & 40 & $100 \%$ \\
\hline
\end{tabular}

Associated diseases included diabetes mellitus ( 8 patients), hypertension (6 patients), coronary heart disease (10 patients) and of the 20 patients tested for thyroid disease 5 patients had hypothyroidism.

Patients had a mild inflammatory syndrome, mean erythrocyte sedimentation rate (ESR) $27 \pm 22(\mathrm{~mm} / \mathrm{h})$ and mean C-reactive protein 3.25 $\pm 8.34(\mathrm{mg} / \mathrm{dl})$.

\section{DISCUSSIONS}

We evaluated symptomatic patients with evidence of CPPD in synovial fluid and/or imaging.

CPPD presents in various clinical forms, but a lot of cases are asymptomatic (2). Louthrenoo et al. described in a study of 91 Thai patients acute monoarthritis and oligoarthritis as the most frequent clinical

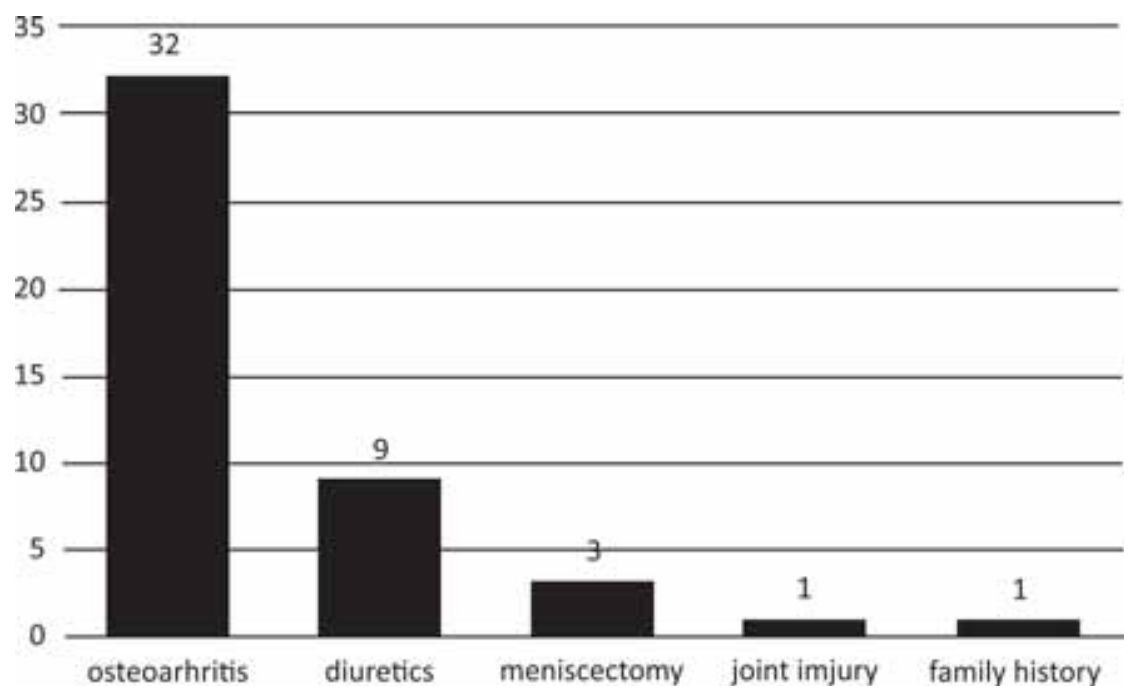

FIGURE 2. The distribution of patients by risk factors 
manifestation and the knee the most common joint involved (17). In our study, the most frequent type of clinical presentation was acute arthritis, representing $70 \%$ of patients. The other two forms of chronic arthritis according to EULAR, chronic CPP crystal inflammatory arthritis and osteoarthritis with CPPD, were difficult to differentiate because $80 \%$ of the patient had underlying osteoarthritis.

Abhishek et al. found that the knee was usually involved; in addition, hand and hip were the most affected joints when no knee chondrocalcinosis was found $(18,19)$.

We also reported that the knee was the most affected joint, clinically and ultrasonographically. Moreover, on radiographs, the knee was the joint most frequently affected by chondrocalcinosis and by osteoarthritis as well. The hand was the second joint involved joint. In the absence of the knee involvement, hand and hip were the most frequent in our study too.

Ageing is known to be one of the most important factors in CPPD (2). In our study, the majority of patients were in seven and eight decades, double comparative with patients from five and six decades.

Ryu et al. and Ramonda et al. observed an association between CPPD and female gender. In our study the percentage of females $(80 \%)$ was notably higher $(20,21)$

Joint damage is another risk factor for CPPD, while the risk for chondrocalcinosis is about $20 \%$ in patients with meniscectomy (22). In our study, joint damage was found 4 patients, one with joint trauma and 3 patients with meniscectomy representing 8\% of the patients with CPPD.

Rho et al. conducted a study in patients with pseudogout and found that loop diuretics were associated with increased risk to develop of the disease. Nine of our patients (18\%) used diuretics, but $77 \%$ of them use thiazide and 33\% loop diuretics (3).

Alcohol consumption and renal insufficiency were not associated with pseudogout in Rho's study (3). In our study no patient recognized alcohol consumption and no patient had renal insufficiency, either.

Hypercalcemia, hyperparathyroidism, hypophosphatasia and hemochromatosis were not demonstrat- ed by Louthrenoo et al. in their study on CPPD patients (17). When evaluating metabolic diseases associated with CPPD, we found hipophosphatasia in one patient and hypomagnesaemia in three patients. Two of the patients with hypomagnesaemia were on diuretics. However, we did not find any patients with hypercalcemia.

Alkaptonuria is a rare genetic disease due to the lack of a specific enzyme, which leads to accumulation of homogentisic acid in the tissue. The most affected areas in the world are Slovakia and Dominican Republic. We are not aware of any studies regarding the prevalence of alkaptonuria in Romania. There is a Slovak minority in Western part of Romania, near the Hungarian border. In our study all patients were from the North-Western part, Transylvania region and screening was negative as expected in all patients tested. Chondrocalcinosis can appear in patients with alkaptonuria and even participate in the ochronotic arthropathy pathogenesis. Screening for alkaptonuria could be considered in the evaluation of CPPD patients because of the simple, inexpensive test used, which is urine alkalinization with $\mathrm{NaOH}(23-25)$.

After analyzing the associated diseases, we found five patients with hypothyroidism. Although is a frequent finding in patients with CPPD, large studies didn't found an association between thyroid disease and chondrocalcinosis $(26,27)$.

There are several limitations of the study, i.e. the small number of patients enrolled and the absence of synovial fluid analysis in some patients.

\section{CONCLUSIONS}

In conclusion, the knee is the most affected joint in CPPD both clinically and by imaging examination. Hip involvement may be found in patients without knee involvement. Acute arthritis is the most frequent form of presentation. Ageing and osteoarthritis are frequent risk factors for CPPD. Although secondary causes of CPPD are rare, screening is very important and is made with simple, inexpensive tests. 


\section{REFERENCES}

1. McCarty D.J. Calcium pyrophosphate dihydrate (CPPD) crystal deposition disease - nomenclature and diagnostic criteria. Ann Int Med. 1977; 87:240-242.

2. Zhang W., Doherty M., Bardin T., et al. European League Against Rheumatism recommendations for calcium pyrophosphate deposition. Part I: Terminology and diagnosis. Ann Rheum Dis. 2011; 70:563-570

3. Rho Y.H., Zhu Y., Zhang Y., et al. Risk factors for pseudogout in the general population. Rheumatology. 2012;51:2070-2074.

4. Neame R.L., Carr A.J., Muir K., et al. UK community prevalence of knee chondrocalcinosis: evidence that correlation with osteoarthritis is through a shared association with osteophyte. Ann Rheum Dis. 2003; 62(6):513-31.

5. Abhishek A. Calcium pyrophosphate deposition disease: a review of epidemiologic findings. Curr Opin In Rheumatol. 2016; 28(2):133-9.

6. Alexander G.M., Dieppe P.A., Doherty M., et al.

Pyrophosphate arthropathy: a study of metabolic associations and laboratory data. Ann Rheum Dis. 1982;41(4):377-81.

7. Abhishek A., Doherty M. Epidemiology of calcium pyrophosphate crystal arthritis and basic calcium phosphate crystal arthropathy. Rheum Dis Clin North Am. 2014; 40(2):177-91.

8. Richette P., Bardin T., Doherty M. An update on the epidemiology of calcium pyrophosphate dihydrate crystal deposition disease. Rheumatology (Oxford). 2009; 48(7):711-5.

9. Swan A., Amer H., Dieppe P. The value of synovial fluid assays in the diagnosis of joint disease: a literature survey. Ann Rheum Dis. 2002; 61(6):493-498.

10. Paparo F., Fabbro E., Ferrero G., et al. Imaging studies of crystalline arthritides. Reumatismo. 2012; 63(4):263-275.

11. Steinbach L.S. Calcium pyrophosphate dihydrate and calcium hydroxyapatite crystal deposition diseases: imaging perspectives. Radiol Clin North Am. 2004; 42(1):185-205, vii.

12. Resnick D., Niwayama G., Goergen T.G., et al. Clinical, radiographic and pathologic abnormalities in calcium pyrophosphate dihydrate deposition disease (CPPD): pseudogout. Radiology. 1977; 122:1-15.

13. Frediani B., Filippou G., Falsetti P., et al. Diagnosis of calcium pyrophosphate dihydrate crystal deposition disease: ultrasonographic criteria proposed. Ann Rheum Dis. 2005; 64(4):638-640.

14. Filippou G., Frediani B., Gallo A., et al. A "new" technique for the diagnosis of chondrocalcinosis of the knee: sensitivity and specificity of high-frequency ultrasonography. Ann Rheum Dis. 2007; 66(8): 1126-1128.

15. Zhang W., Doherty M., Pascual E., et al. EULAR recommendations for calcium pyrophosphate deposition. Part II: Management. Ann Rheum Dis. 2011; 70:571-575.

16. McMullan P., McCarthy G. Treatment and management of pseudogout: insights for the clinician. Ther Adv in Musculoskelet Dis. 2012; 4(2):121-31

17. Louthrenoo W., Sukitawut. Calcium pyrophosphate dihydrate crystal deposition: a clinical and laboratory analysis of 91 Thai patients. J Med Assoc Thai. 1999; 82(6):569-76.

18. Abhishek A., Doherty S., Maciewicz R, et al. Chondrocalcinosis frequently occurs at the wrists and hips in the absence of knee involvement. Ann Rheum Dis. 2013; 71(Suppl 3):S442.

19. Abhishek A., Doherty S, Maciewicz R., et al. Chondrocalcinosis is common in the absence of knee involvement. Arthritis Res Ther 2012; 14: R205.

20. Ramonda R., Musacchio E., Perissinotto E., et al. Prevalence of chondrocalcinosis in Italian subjects from northeastern Italy. The Pro. V. A. (PROgetto Veneto Anziani) study. Clin Exp Rheumatol. 2009; 27:981-4.

21. Ryu K., Iriuchishima T., Oshida M. et al. The prevalence of and factors related to calcium pyrophosphate dihydrate crystal deposition in the knee joint. Osteoarthritis Cartilage. 2014; 22(7):975-9.

22. Doherty M., Watt I., Dieppe PA. Localized chondrocalcinosis in postmeniscectomy knees. Lancet. 1982; 1:1207-10.

23. Zatkova A. An update on molecular genetics of Alkaptonuria (AKU).J Inherit Metab Dis. 2011; 34(6), 1127-36.

24. McClure J., Smith P.S., Gramp A.A. Calcium pyrophosphate dihydrate (CPPD) deposition in ochronotic arthropathy. J ClinPathol. 1983; 36: 894-902.

25. Rynes R.I., Sosman J.L., Holdsworth D.E. Pseudogout in ochronosis. Arthritis Rheum. 1975; 18: 21-25.

26. Chaisson C.E., McAlindon T.E., Felson D.T, et al. Lack of association between thyroid status and chondrocalcinosis or osteoarthritis: the Framingham Osteoarthritis Study. J Rheumatol. 1996; 23(4):711-5.

27. Job-Deslandre C., Menkes C.J., Guinot M., et al. Does hypothyroidism increase the prevalence of chondrocalcinosis? Br J Rheumatol. 1993 Mar; 32(3):197-8. 